\title{
Quiste dentígero en niños
}

Fernanda Astrogilda de Sousa Feitosa ${ }^{1}$, Amanda Simião Figueiredo ${ }^{1}$, Carolina Carvalho de Oliveira Santos ${ }^{1}$ Bertha Angélica Chávez Gonzalez², Raquel Gonçalves Vieira-Andrade ${ }^{1}$ Thiago Fonseca-Silva ${ }^{1}$

\section{Resumen}

Introducción: El quiste dentígero es el segundo tipo de quistes odontogénicos más frecuentes delos maxilares, desarrollándose a partir de alteraciones del epitelio del órgano del esmalte, donde ocurre una acumulación de líquido entre el epitelio y el esmalte. Por lo general está relacionado a la corona de un diente no erupcionado. Objetivos: el objetivo de este estudio fue analizar los aspectos de diagnóstico y tratamiento de los quistes dentígero en pacientes pediátricos. Métodos: se realizó una búsqueda de artículos científicos indexados en la base de datos bibliográficos PUBMED ${ }^{\circledR}$ en el período comprendido entre febrero y marzo de 2015, mediante el descriptor "dentigerous cyst". Resultados: Se encontraron 147 artículos publicados en los últimos 10 años. Todos los artículos que trataban sobre quiste dentígero en niños fueron incluidos en este estudio. La literatura revisada destaca puntos importantes sobre la epidemiología, manejo y tratamiento de quiste dentígero en pacientes pediátricos. Conclusión: De los artículos revisados se puede concluir que el quiste dentígero es más frecuente en los niños del género masculino y con una edad media de 10 años. Afecta principalmente a la mandíbula y tanto la enucleación como la marsupialización están indicados para el tratamiento de quistes.

Palabras claves: Quiste dentígero, odontopediatría; quiste odontogénico.

\section{Artigo de Revisão}

\section{Cisto dentígero na infância}

\section{Resumo}

Introdução: $\mathrm{O}$ cisto dentígero é o segundo tipo de cisto odontogênico mais frequente dos maxilares, desenvolvendo-se a partir de alterações do epitélio reduzido do órgão do esmalte, onde ocorre acúmulo de líquido entre esse epitélio e o esmalte dentário. Normalmente está relacionado à coroa de um dente não irrompido. $\mathbf{O b -}$ jetivos: o propósito deste trabalho foi discutir os aspectos de diagnostico e manejo de cistos dentígeros em pacientes infantis. Métodos: Foi

1. Faculdade de Odontologia; Faculdade Leão Sampaio - Juazeiro do Norte, Ceará, Brasil.

2. Instituto Nacional Materno Perinatal, Lima, Perú. 
realizada uma busca de artigos científicos indexados na base bibliográfica PUBMED® no período entre fevereiro e março de 2015, com a utilização do descritor "dentigerous cyst". Resultados: foram encontrados 147 artigos publicados nos últimos 10 anos. Todos os trabalhos que que abordavam cisto dentígero em crianças foram incluídos nesse estudo. A literatura revisada, destaca pontos importantes sobre a epidemiologia, manejo e tratamento de cisto dentígero em pacientes infantis. Conclusão: A partir dos artigos revisados pode-se concluir que o cisto dentígero é mais prevalente em crianças do gênero masculino, com idade média de 10 anos. Acomete principalmente a mandíbula e tanto a enucleaçao como a marsupialização são indicados para o tratamento dos cistos.

Palavras chaves: Cisto dentígero, odontopediatria, cisto odontogênico.

\section{Review Article}

\section{Dentigerous cyst in children}

\section{Abstract}

Introduction: The dentigerous cyst is the second most frequent odontogenic cyst of the jaws, developing from changes in the reduced epithelium of the enamel organ, due to accumulation of fluid between the epithelium and the tooth enamel. Usually it relates to the crown of a non erupted tooth. Objectives: the purpose of this study was to discuss aspects of diagnosis and management of dentigerous cysts in children. Methods: the search of scientific articles indexed in bibliographic database PUBMED® in the period between February and March 2015, using the descriptor "dentigerous cyst" was performed. Results: 147 articles published in the last 10 years were found. All papers that addressed dentigerous cyst in children were included in this study. The articles reviewed highlighted important issues regarding epidemiology, management and treatment of dentigerous cyst in pediatric patients. Conclusion: the dentigerous cyst is more prevalent in males with a mean age of 10 years, mainly affecting the mandible. Both enucleation and marsupialization are indicated for the treatment of cysts.

Key words: Dentigerous cyst, pediatric dentistry, odontogenic cyst.

\section{Introducción}

El Quiste dentígero (QD) es el segundo tipo más común de quiste odontogénico, comprendiendo cerca del $20 \%$ de todos los quistes encontrados en los maxilares. Su origen está relacionado con acúmulo de líquido entre o epitelio reducido del órgano do esmalte e la corona de un dente no irrumpido. ${ }^{1-5}$

La etiopatogénesis de esa lesión aún no es clara, ${ }^{3-11}$ sin embargo, algunos autores sugieren que procesos inflamatorios locales pueden estar asociados al origen del quiste. . $-8,12,13^{-13}$

Radiográficamente el QD se manifiesta como una área radiolúcida unilocular bien definida asociada a la corona de un diente no erupcionado. 1,3,5,6,9,11 
Resorciones óseas, expansión de corticales y dislocamientos dentarios son eventos comúnmente asociados a los quistes dentígeros. Los métodos empleados en el tratamiento del quiste dentígero incluyen la marsupialización, enucleación y/o curetaje. . $^{2,5,6,8,13}$

Quistes dentígeros en niños son poco frecuentes. La frecuencia de ese tipo de quiste en la infancia es de 4 a 9\% de los casos. ${ }^{9,10,11}$ El tratamiento del QD en niños es aún bastante discutido en la literatura, en vista de la posibilidad de mantener o no el diente afectado.6 El propósito de este trabajo es, a través de una revisión de literatura, discutir los aspectos de diagnósticos y tratamientos de los quistes dentígeros en niños.

\section{Metodología}

La metodología adoptada en el presente estudio consistió en una búsqueda bibliográfica de artículos científicos indexados en la base bibliográfica PUBMED®, en el mes de marzo del 2015, con las palabras-claves "dentigerous cyst". Los criterios de inclusión para la selección de los trabajos, consistieron en artículos en la lengua inglesa publicados en los últimos 10 años (del 2005 al 2015). Fueron identificados 147 artículos como potencialmente relevantes. Los artículos que abordaban manejo y tratamiento de quiste dentígero en niños fueron incluidos en el estudio. El total de 19 artículos fueron seleccionados para ser parte del trabajo.

\section{Revisión de la literatura}

\section{Patogénesis}

La etiología del quiste dentígero aún es desconocida, se cree que el quiste es formado a partir del acumulo del fluído entre el epitelio reducido del esmalte y la corona del diente. ${ }^{2,6-9}$ El acumúlo del fluido parece ser resultado de la presión ejercida por el diente en erupción en el folículo peri coronario (epitelio reducido del órgano del esmalte), obstruyendo así el flujo venoso y generando el acumulo de líquidos y formación cística. Existe también la hipótesis de que el quiste dentígero se desenvuelva a partir de la proliferación de células del folículo luego de la erupción impedida. ${ }^{12}$ Esa proliferación celular resulta en un aumento de la tensión osmótica, y consecuentemente, ocurre la formación del quiste dentígero ${ }^{8}$. Otra hipótesis para la etiopatogenesis del QD es la inflamación periapical proveniente de dientes deciduos no vitales. ${ }^{7-13}$ El proceso inflamatorio periapical estimularía el folículo del diente permanente no erupcionado, lo que resultaría en la formación del quiste dentígero. ${ }^{8,9,14}$

\section{Características Clínicas e Radiográficas}

El quiste dentígero es el segundo tipo de quiste más común de los quistes de desenvolvimiento maxilo-mandibulares, representando cerca de 20 a $24 \%$ de los quistes del sistema estomatognático. ${ }^{6,5}$ Clínicamente este quiste se presenta con una lesión cística asociado a un diente incluido. ${ }^{8}$ Los dientes más afectados por esta patología son terceros molares inferiores, caninos e premolares inferiores. ${ }^{3,6,8}$ Dientes supernumerarios también pueden esrat afectados y comprenden de 5 a $6 \%$ de los quistes dentígeros. ${ }^{10}$ Algunos autores sugieren que esta condición es más común en la segunda es tercera década de vida, siendo considerada una patología rara en niños. ${ }^{9}$

En cuanto a la sintomatología, el quiste dentígero generalmente es asintomático. No son comunes dolor ni incomodidad, y estos pueden estar relacio- 
nados a la infección secundaria del quiste. ${ }^{15} \mathrm{El}$ retardo de la erupción dentaria puede ser un indicador de la presencia del quiste. ${ }^{3,12}$ Debido a la ausencia del sintomatología, el quiste puede alcanzar grandes proporciones, resultando en resorción ósea compresiva y/o expansión de la cortical ósea. ${ }^{3,5,11}$ Complicaciones como fractura patológica del hueso comprometido, infección secundaria, dislocamientos dentarios, movilidad dentaria y deformaciones faciales y oseas también son aspectos relacionados a los quistes dentígeros. ${ }^{3,14}$

Normalmente, los quistes dentígeros son descubiertos en exámenes radiográficos de rutina. Radiográficamente esos quistes se presentan como una área radiolucida unilocular bien definida, algunas veces con la presencia de bordes radiopacas compatible con esclerosis ósea, en torno de la corona de un diente incluido. ${ }^{1,3,6,14}$ Características radiográficas adicionales incluyen dislocamiento del canal mandibular, resorción de la pared de este canal, resorción radicular de dientes permanentes adyacentes y la posibilidad de fractura patológica. ${ }^{15-17}$

\section{Diagnóstico}

El diagnóstico del quiste dentígero es realizado a partir de un minucioso examen clínico y radiográfico, así como del análisis histopatológico. 1,3,6,8,10,11,18 Histopatológicamente, el QD presenta una cápsula de tejido conjuntivo fibroso fijado débilmente, revestida por epitelio en general, del tipo pavimentos estratificado no queratinizado. Epitelio pavimentoso hiperplásico y epitelio reducido del esmalte también pueden revestir a lesión. En la cápsula se puede observar la presencia de un infiltrado inflamatorio de tipo mononuclear y pequeñas islas o cordones de restos de epitelio odontogênico. ${ }^{3}$
El quiste periapical, queratoquiste odontogénico, granuloma central de células gigantes y ameloblastoma unicístico hacen diagnóstico diferencial con QD. ${ }^{3}$ La punción aspiratoria es una maniobra auxiliar al diagnóstico, la cual hace la evaluación del contenido y coloración de la substancia en el interior del quiste. ${ }^{3,14}$

\section{Tratamiento}

El tratamiento del quiste dentígero depende del tamaño, localización y configuración de la lesión. La literatura describe que el tratamiento del quiste dentígero puede ser realizado por medio de la marsupialización o enucleación. ${ }^{3,5,6,8,14}$ La marsupialización es un procedimiento conservador que consiste en establecer una comunicación entre el ambiente interno del quiste y la cavidad bucal. Ese procedimiento posibilita el equilibrio de la presión hidrostática intralesión, y consecuentemente, descompresión y reducción del quiste, posibilitando la neoformación del tejido óseo, reduciendo así el riesgo de ocurrencia de grandes defectos óseos y parestesia. ${ }^{9}$ La enucleación consiste en la remoción completa de la lesión así como del diente asociado. ${ }^{1,3,5}$

\section{Discusión}

A partir del análisis de los artículos, fue observado que las lesiones del QD fueron encontradas en niños de ambos géneros, siendo más prevalentes en niños del género masculino (72,3\%). La rango de edad de los individuos varia de 01 a 12 años de edad (mediana = 10 años), y el sitio afectado más prevalente fue la mandíbula $(83,3 \%)$ (Tabla 1). Por lo tanto, conocer los datos de prevalencia e incidencia de QD en niños es de suma importancia para el estabelecimiento de protocolos y acompañamiento de esas lesiones, además de posibilitar mejores 


\begin{tabular}{|c|c|c|c|c|c|c|c|c|}
\hline Autor & Año & $\begin{array}{c}\text { Casos } \\
\text { Relatados }\end{array}$ & Género & $\begin{array}{l}\text { Edad } \\
(\text { años })\end{array}$ & Local & $\begin{array}{l}\text { Exámenes } \\
\text { Realizados }\end{array}$ & Tratamiento & $\begin{array}{l}\text { Extracción } \\
\text { dentária }\end{array}$ \\
\hline $\begin{array}{l}\text { Kalaskar RR } \\
\text { y Cols }{ }^{16}\end{array}$ & 2007 & 1 & Masculino & 7 & Maxilar & $\begin{array}{c}\text { Clínicos, Radiográficos, } \\
\text { Histológico }\end{array}$ & Enucleación & $\mathrm{Si}$ (diente 11) \\
\hline Passi S y Cols ${ }^{17}$ & 2008 & 1 & Masculino & 10 & Mandíbula & $\begin{array}{c}\text { Clínicos, Radiográficos, } \\
\text { Histológico }\end{array}$ & Marsupialización & $\begin{array}{c}\mathrm{Si} \\
\text { (dientes } 83,84 \mathrm{e} 85 \text { ) }\end{array}$ \\
\hline $\begin{array}{c}\text { Gondim JO } \\
\mathrm{y} \mathrm{Cols}^{13}\end{array}$ & 2008 & 1 & Masculino & 4 & Maxilar & $\begin{array}{c}\text { Clínicos, Radiográficos, } \\
\text { Histológico }\end{array}$ & Marsupialización & $\mathrm{Si}$ (diente 51) \\
\hline $\begin{array}{l}\text { Kamasaky Y } \\
\text { y Cols }^{1}\end{array}$ & 2009 & 1 & Masculino & 12 & Mandíbula & $\begin{array}{l}\text { Clínicos, Radiográficos, Punción } \\
\text { aspiratória, Histológico }\end{array}$ & Marsupialización & $\begin{array}{c}\mathrm{Si} \\
(\text { dientes } 73,74 \text { y } 75)\end{array}$ \\
\hline \multirow{2}{*}{$\begin{array}{l}\text { Shivaprakash P } \\
\text { y Cols }{ }^{6}\end{array}$} & \multirow{2}{*}{2009} & 1 & Femenino & 10 & Mandíbula & $\begin{array}{l}\text { Sangre, Clínicos, } \\
\text { Radiográficos e } \\
\text { Histopatológico }\end{array}$ & $\begin{array}{l}\text { Enucleación y } \\
\text { Marsupialización }\end{array}$ & Si (dientes 75 y 85 ) \\
\hline & & 1 & Masculino & 10 & Mandíbula & $\begin{array}{c}\text { Clínicos, Radiográficos, } \\
\text { Histológico }\end{array}$ & Marsupialización & Si (diente 75) \\
\hline $\begin{array}{l}\text { Yahara Y } \\
\text { y Cols }{ }^{2}\end{array}$ & 2009 & 21 & $\begin{array}{l}12 \text { Masculino } \\
09 \text { Femenino }\end{array}$ & 2 a 10 & Mandíbula & $\begin{array}{c}\text { Clínicos, Radiográficos, } \\
\text { Histológicos }\end{array}$ & Marsupialización & No especificado \\
\hline $\begin{array}{l}\text { Sharma D } \\
\text { y Cols }^{7}\end{array}$ & 2010 & 1 & Masculino & 12 & Maxilar & $\begin{array}{l}\text { Clínicos, Radiográficos, } \\
\text { Histopatológicos }\end{array}$ & Enucleación & $\begin{array}{c}\mathrm{Si} \\
\text { (supernumerarios) }\end{array}$ \\
\hline \multirow{2}{*}{$\begin{array}{l}\text { Kirtaniya BC } \\
\text { y Cols }^{3}\end{array}$} & \multirow[b]{2}{*}{2010} & 1 & Masculino & 7 & Mandíbula & $\begin{array}{l}\text { Clínico, Sangre, Radiográficos, } \\
\text { Punción Aspirativa, Histológico }\end{array}$ & Marsupialización & $\mathrm{Si}$ (dientes 74 y 75 ) \\
\hline & & 1 & Masculino & 10 & Mandíbula & $\begin{array}{c}\text { Clínico, Sangre, } \\
\text { Radiográficos, Punción } \\
\text { Aspirativa, Histológico }\end{array}$ & Marsupialización & $\mathrm{Si}$ (diente 83) \\
\hline $\begin{array}{l}\text { Pramod DSR Y } \\
\text { Shukla JV }^{8}\end{array}$ & 2011 & 1 & Masculino & 7 & Maxilar & $\begin{array}{l}\text { Clínicos, Sangre, Radiográficos, } \\
\text { Punción Aspirativa }\end{array}$ & Enucleación & Si (diente 23) \\
\hline $\begin{array}{l}\text { Suresh R y } \\
\quad \text { Cols }^{5}\end{array}$ & 2011 & 1 & Masculino & 1 & Mandíbula & $\begin{array}{l}\text { Clínicos, Radiográficos, To- } \\
\text { mografía Histopatológico }\end{array}$ & Enucleación & Si (diente 36) \\
\hline $\begin{array}{l}\text { Tilakraj TN } \\
\text { y Cols }{ }^{9}\end{array}$ & 2011 & 1 & Masculino & 4 & Maxilar & $\begin{array}{c}\text { Clínicos, Radiográficos, } \\
\text { Punción Aspirativa, Biópsia, } \\
\text { Histopatológico }\end{array}$ & Enucleación & $\mathrm{Si}$ (diente 54) \\
\hline $\mathrm{Hu} \mathrm{YH}$ y Cols ${ }^{4}$ & 2011 & 1 & Masculino & 10 & Mandíbula & $\begin{array}{l}\text { Clínicos, Radiográficos, } \\
\text { Biópsia, Histopatológico, }\end{array}$ & Marsupialización & $\mathrm{Si}$ (dientes 74 e 75 ) \\
\hline $\begin{array}{l}\text { Oliveira LSAF } \\
\text { y Cols }^{15}\end{array}$ & 2012 & 1 & Masculino & 3 & Maxilar & $\begin{array}{l}\text { Clínicos, Radiográficos, } \\
\text { Punción aspiratória, } \\
\text { Histológico }\end{array}$ & Enucleación & $\mathrm{Si}$ (dientes 12 y 13 ) \\
\hline Agrawal NK ${ }^{10}$ & 2012 & 1 & Masculino & 11 & Maxilar & $\begin{array}{l}\text { Clínicos, Radiográficos, } \\
\text { Tomografía, Biópsia, } \\
\text { Histopatológico }\end{array}$ & Enucleación & $\mathrm{Si}$ (supernumerarios) \\
\hline $\begin{array}{c}\text { Lima EM } \\
\text { y Cols }\end{array}$ & 2013 & 1 & Masculino & 10 & Maxilar & $\begin{array}{l}\text { Clínicos, Radiográficos, } \\
\text { Histopatológico }\end{array}$ & Enucleación & $\begin{array}{c}\mathrm{Si} \\
\text { (dientes } 51,52 \text { y } 53)\end{array}$ \\
\hline $\begin{array}{l}\text { Hegde y Cols } \\
\mathrm{RJ}^{14}\end{array}$ & 2013 & 1 & Masculino & 9 & Mandíbula & $\begin{array}{l}\text { Clínicos, Radiográficos, Punción } \\
\text { Aspirativa, Histopatológico, }\end{array}$ & Enucleación & $\mathrm{Si}$ (dientes 35 y 75 ) \\
\hline $\begin{array}{l}\text { Wiemer SJ } \\
\text { y Cols }{ }^{19}\end{array}$ & 2013 & 1 & Masculino & 8 & Mandíbula & $\begin{array}{l}\text { Clínicos, Radiográficos, } \\
\text { Tomografía, Biópsia, } \\
\text { Histopatológico }\end{array}$ & Enucleación & $\mathrm{Si}$ (dientes 84 y 85$)$ \\
\hline Issar Y y Cols ${ }^{18}$ & 2014 & 1 & Masculino & 11 & Maxilar & $\begin{array}{l}\text { Clínicos, Sangre, Radiográficos, } \\
\text { Tomografía, Punción Aspirativa, } \\
\text { Biópsia, Histopatológico }\end{array}$ & Enucleación & $\mathrm{Si}$ (dientes 13 y 14$)$ \\
\hline
\end{tabular}

Tabla 1. Presentación de los datos extraídos de los artículos revisados. 
condiciones para el diagnóstico y tratamiento de las mismas. No obstante, hay una escasez de trabajos científicos que aborden esa temática. ${ }^{5,11}$

Son escasos los trabajos que relatan el manejo del quiste dentígero en pacientes pediátricos. Algunos autores creen que la marsupialización es la mejor elección para el tratamiento del QD en niños. De los artículos investigados, el 75\% de los casos fueron tratados con la marsupialización. Los autores consideran que esa modalidad de tratamiento es una opción conservadora, que además de preservar estructuras importantes, favorece la erupción de los elementos dentarios en formación o el tracionamiento ortodóntico del mismo. ${ }^{1,3-5}$ Sin embargo, vale resaltar que ese manejo no es un consenso en literatura. Existen autores que creen que la enucleación es también la mejor forma de tratamiento en pacientes infantiles por minimizar significativamente las tasas de recidiva. . $^{511,14,15,18}$

Varios autores corroboran que durante la terapia cirúgica para el tratamiento del $\mathrm{QD}$, los dientes deciduos asociados a las lesiones necesitan ser removidos. 13,4,6,9,11,14,19 En relación a los dientes permanentes asociados al QD, 25\% de los casos analizados fueron tratados por medio de la enucleación de la lesión y remoción del diente asociado., $5,14,17$ Diversos autores discuten que la remoción de los dientes envueltos por el QD, cuando es viable la erupción, se traduce en una terapia radical e invasiva. Sin embargo, esa modalidad de tratamiento garantiza la remoción completa de la lesión, evitando así que remanentes de epitelio cístico sean sepultados in vivo, lo que reduciría significativamente las tasas de recidiva. ${ }^{5,11}$ Por otro lado, estudios mostraron que la marsupialización, además de reducir significativamente el tamaño del quiste, estimula la erupción de elementos dentarios permanentes envueltos por la lesión. ${ }^{1-4,6}$

En los trabajos analizados, en ambas las terapias empleadas para el tratamiento de QD, el seguimiento de los pacientes variaran entre 06 meses a 04 años de acompañamiento. Durante ese período no fueron observadas recidivas de QD ni ninguna otra condición patológica en la área anteriormente afectada por la lesión. El pronóstico para el tratamiento del QD en niños ha sido bastante favorable, independientemente de la técnica utilizada. Sin embargo, los criterios para la elección de una de estas modalidades de tratamiento aún no son claramente definidos.

\section{Conclusión}

A partir de los artículos revisados se puede concluir que el quiste dentígero es más prevalente en niños del género masculino, con edad media de 10 años, afectando principalmente la mandíbula. Tanto la enucleación como la marsupialización son indicados para el tratamiento de los quistes dentígeros.

\section{Referencias bibliográficas}

1. Kamasaki Y; Sasaki Y, Fujiwara T. Management of an extensive dentigerous cyst in a 12-year-old boy. Pediatr Dent J. 2009; 19: 234-9.

2. Yahara Y, Kubota Y, Yamashiro T, Shirasuna, K. Eruption prediction of mandibular premolars associated with dentigerous cysts. Oral Surg Oral Med Oral Pathol Oral Radiol Endod. 2009; 108:28-31.

3. Kirtaniya BC, Sachdev V, Singla A, Sharma AK. Marsupialization: A conservative approach for treating dentigerous cyst in children in the mixed dentition. J Indian Soc Pedod Prev Dent. 2010; 28:203-8. 
4. Hu YH, Chang Y, Tsai A. Conservative treatment of dentigerous cyst associated with primary teeth. Oral Surg Oral Med Oral Pathol Oral Radiol Endod. 2011, 112:e5-e7.

5. Suresh R, Janardhanan M, Joseph AP, Vinodkurmar RB, Peter S. A Rare Case of Dentigerous Cyst in a One Year Old Child: The Earliest Known Reported Occurrence. Head and Neck Pathol. 2011; 5:171- 4.

6. Shivaprakash P, Rizwanullah T, Baweja DK, Noorani H. Save-a-tooth: Conservative surgical management of dentigerous cyst. J Indian Soc Pedod Prevent Dent. 2009; 27:52-7.

7. Sharma D, Garg S, Singh G, Swami S. Trauma-induced dentigerous cyst involving an inverted impacted mesiodens: case report. Dent Traumatol. 2010; 26: 289-91.

8. Pramod DSR, Shukla JV. Dentigerous cyst of maxilla in a young child. Natl J Maxillofac Surg. 2011; 2:196-9.

9. Tilakraj T N, Kiran NK, Mukunda KS, Shwetha R. Non syndromic unilateral dentigerous cyst in a 4-year-old child: A rare case report. Contemp Clin Dent. 2011; 2:398-401.

10. Agrawal NK. Dentigerous cyst in a child associated with multiple inverted supernumerary teeth: A rare occurrence. Int J Burn Trauma. 2012; 2:171-3.

11. Lima ENA, Maia CADM, Gurgel AC, Oliveira PT, Medeiros AMC. Conservative management of dentigerous cyst in a child. Int J Pediatr Otorhinolaryngol. 2013; 8: e1-e4.

12. Narang RS, Machanda AS, Arora P, Randhawa K. Dentigerous cyst of inflammatory origin-a diagnostic dilema. Ann Diagn Pathol. 2012; 16: 119-23.

13. Gondim JO, Moreira Neto JJS, Nogueira RLM, Giro EMA. Conservative management of a dentigerous cyst secondary to primary tooth trauma. Dent Traumatol. 2008; 24:676-9.

14. Hegde RJ, Khare SS, Devrukhkar VN. Dentigerous Cyst in a young child: Clinical Insight and A Case report. J Indian Soc Pedod Prev Dent. 2013; 31:209-11.

15. De Andrade Freitas Oliveira LS, Souza DO, Neves FS, dos Santos JN, Campos PS, Crusoé-Rebello I. Uncommon dentigerous cyst related to a maxillary lateral incisor in a 03-year-old boy. Oral Maxillofac Surg. 2012; 16 : 383-8.

16. Kalaskar R R, Tiku A, Damle S G. Dentigerous cysts of anterior maxilla in a young child: A case report. J Indian Soc Pedod Prev Dent. 2007; 25:187-90.

17. Passi S, Gauba k, Agnihortri A, Sharma R. Dentigerous cyst in primary dentition: a case report. J Indian Soc Pedod Prev Dent. 2008; 26: 168-70.

18. Issar Y, Kaushal N, Goomer P. Unusual case of concomitant occurrence of Tessier's number 7 cleft and dentigerous cyst. Contemp Clin Dent. 2014; 5:402-5.

19. Wiemer SJ, Pruitt CA, Rallis DJ, Viozzi CF. Use of a Modified Removable Partial Denture as a Marsupialization Stent in a Pediatric Patient. J Oral Maxillofac Surg. 2013, 71:1382-1386.

Recibido: 08-09-2015

Aceptado: 19-01-2016

Correspondencia Thiago Fonseca-Silva, Email: thiagofonsecasilva@gmail.com.

Faculdade de Odontologia Leão Sampaio-CE. Avenida Maria Leticia Leite Pereira s/n -

Juazeiro do Norte-CE CEP: 63180-000TEL: +55 8821011046 